\title{
Imagens e representações sociais: a fotolinguagem e photovoice na produção de dados sobre fenômenos de saúde
}

\author{
Images and social representations: photolanguage and photovoice in data production on health \\ phenomena \\ Imágenes y representaciones sociales: fotolenguaje y fotovoz em la producción de datos sobre \\ fenómenos de salud
}

\author{
Nathália Nunes Gomes ${ }^{1}$ (C) \\ Julyana Gall da Silva ${ }^{1}$ (]) \\ Ana Beatriz Azevedo Queiroz ${ }^{1}$ (D) \\ Márcia de Assunção Ferreira ${ }^{1}$ (1) \\ Thémis Apostolidis² \\ Rafael Celestino da Silva ${ }^{1}$ (1)
}

\begin{abstract}
1. Universidade Federal do Rio de Janeiro, Escola de Enfermagem Anna Nery. Rio de Janeiro, RJ, Brasil.
\end{abstract}

2. Aix-Marseille Université, Laboratoire de Psychologie sociale. Aix-en-Provence, France

Autor correspondente:

Rafael Celestino da Silva.

E-mail: rafaenfer@yahoo.com.br

Recebido em 02/08/2021.

Aprovado em 06/09/2021.

DOI:https://doi.org/10.1590/2177-9465-EAN-2021-0291

\section{RESUMO}

Objetivo: refletir sobre o uso de fotos na apreensão das representações sociais de fenômenos na área da saúde, a partir da aplicação da técnica da fotolinguagem e do método photovoice. Método: estudo teórico-reflexivo desenvolvido em duas etapas: de análise da literatura; e análise reflexiva sobre a técnica e o método aplicados, pautados nas representações sociais. Desenvolvimento: a análise apontou que as fotos possibilitam captar a zona muda das representações, a dimensão dos afetos, dos símbolos e são parte da estratégia de triangulação metodológica para entendimento das múltiplas dimensões que concorrem para a organização do campo da representação social de fenômenos da área da saúde. Conclusão e implicações para a prática: as fotos potencializam a apreensão da dimensão figurativa da representação, essencial na compreensão da elaboração do pensamento social. O uso da fotolinguagem e do photovoice na prática operacional de pesquisa contribui para o avanço do conhecimento nos estudos de representações sociais na área da saúde.

Palavras-chave: Coleta de dados; Fotografia; Pesquisa em Enfermagem; Pesquisa Qualitativa; Psicologia Social.

\section{ABSTRACT}

Objective: to reflect on the use of photographs in the apprehension of social representations of phenomena in health care by applying the photolanguage technique and the photovoice method. Method: a reflective and theoretical study developed in two stages: literature analysis; and reflective analysis on the technique and method applied, based on social representations. Development: the analysis pointed out that photographs make it possible to capture the silent zone of representations and the dimension of affections, symbols, as well as that they are part of the methodological triangulation strategy to understand the multiple dimensions that contribute to the organization of the field of social representation of phenomena in health care. Conclusion and implications for the practice: photographs enhance apprehension of the figurative dimension of the representation, essential in understanding the elaboration of social thinking. The use of photolanguage and photovoice in the research operational practice contributes to the advancement of knowledge in studies of social representations in health care.

Keywords: Data Collection; Photography; Nursing Research; Qualitative research; Social Psychology.

\section{RESUMEN}

Objetivo: Reflexionar sobre el uso de fotografías en la aprehensión de representaciones sociales de fenómenos en el área de la salud, desde la perspectiva de la aplicación de la técnica del fotolenguaje y del método de la fotovoz. Método: Estudio teórico-reflexivo desarrollado en dos etapas: análisis de la literatura; y análisis reflexivo sobre la técnica y el método aplicados basado en las representaciones sociales. Desarrollo: El análisis señaló que las fotografías permiten captar la zona muda de las representaciones, la dimensión de los afectos, de los símbolos y forman parte de la estrategia de triangulación metodológica para comprender las múltiples dimensiones que contribuyen a la organización del campo de la representación social de fenómenos en el área de la salud. Conclusión e implicaciones en la práctica: Las fotografías intensifican la aprehensión de la dimensión figurativa de la representación, fundamental para comprender la elaboración del pensamiento social. El uso de fotolenguaje y de la fotovoz en la práctica operativa de la investigación contribuye al avance del conocimiento en los estudios de las representaciones sociales en el área de la salud.

Palabras clave: Recolección de datos; Fotografía; Investigación en enfermería; Investigación cualitativa; Psicología social. 


\section{INTRODUÇÃO}

A representação social (RS) é entendida como um conjunto de proposições e explicações que surge na vida cotidiana no curso de comunicações interpessoais. Equivalente, na sociedade atual, aos mitos e sistemas de crenças das sociedades tradicionais; pode também ser vista como a versão contemporânea do senso comum ${ }^{1}$.

A estrutura da RS tem uma dupla natureza: conceptual e figurativa. O conceito é capaz de dar um sentido a um objeto não presente, simbolizá-lo; o percepto significa recuperar esse objeto, dá-lhe concretude, tangibilidade. Portanto, a RS faz compreender a toda figura um sentido e a todo sentido uma figura ${ }^{1}$. Esse processo ocorre por meio da ancoragem e da objetivação, mobilizados pelo indivíduo frente a algo incomum.

A ancoragem busca ancorar ideias estranhas, reduzi-las a categorias e a imagens comuns, colocá-las em um contexto familiar ${ }^{1}$. Tal mecanismo tenta se aproximar das situações desconhecidas, categorizando os fenômenos em classes já conhecidas e, a partir disso, conferindo sentido a eles. A objetivação atribui uma dimensão imagética ao objeto apresentado à compreensão; logo, o que era desconhecido torna-se objetivo, assumindo o contorno de real, com concretude, palpável ${ }^{1}$.

Assim, a objetivação naturaliza a representação, limitando o objeto à imagem mental. A naturalização das noções lhes dá valor de realidades concretas, legíveis e utilizáveis na ação sobre o mundo e os outros. Com isso, a estrutura imagética da $\mathrm{RS}$ se torna guia de leitura, uma teoria de referência para se compreender a realidade ${ }^{1}$.

À luz desses preceitos teóricos, parte-se da premissa de que a apreensão da RS deve abarcar duas faces: a verbal e a imagética. Sob tal ótica, é preciso ampliar as estratégias metodológicas de produção de dados para além daquelas técnicas pautadas em mensagens numéricas e escritas ${ }^{2}$, com vistas a conferir adensamento às análises.

Em face disso, neste artigo se realça o uso das imagens como fonte de dados na pesquisa em RS na área da saúde, em especial, por meio das fotografias. Isto porque, as imagens, quando utilizadas nas pesquisas de RS, favorecem a socialização de significados e são elementos ativos na sua formação, já que a realidade não é definida só por palavras ${ }^{3}$.

No caso dos fenômenos de RS na área da saúde, estudos prévios têm evidenciado essa forte dimensão imagética, como, por exemplo, a tecnologia/cuidados nos setores de terapia intensiva, objetivados na imagem de "um bicho de sete cabeças" para enfermeiros inexperientes; ou as RS da alimentação para os adolescentes, cujos sentidos fazem relação com o peso e a saúde, nos quais ser saudável se estrutura na imagem de um indivíduo magro/"forte"e o gordo objetiva a pessoa não saudável ${ }^{4,5}$.

Em análise exploratória de estudos existentes sobre o uso de fotos para os estudos de RS, em particular, com o emprego da fotolinguagem e do photovoice, técnica e método que são os focos de interesse do presente artigo, foram identificadas três produções sobre o uso da fotolinguagem. Nessa análise, se verificou que a técnica tem sido utilizada como estratégia grupal numa perspectiva terapêutica, e como técnica de coleta de dados em investigações, ilustrada pela pesquisa das RS de idosos em reabilitação sobre a deficiência física ${ }^{6,7}$.

Quanto ao uso do photovoice, destaca-se a revisão que analisou publicações sobre a sua utilização por enfermeiros na educação em saúde. Foram captados 27 estudos, categorizados em duas áreas temáticas: transição saúde-doença e criação de ambientes favoráveis à saúde. Nenhum dos estudos desenvolvidos foi pautado nas $\mathrm{RS}^{8}$.

Essa incipiência de estudos acerca da fotolinguagem e phototovoice na interface com as análises de RS, acrescentada ao fato de que, na experiência dos pesquisadores como avaliadores Ad hoc observam-se fragilidades metodológicas nos estudos de RS, mormente pela falta de coerência na escolha das técnicas de coleta de dados e na elaboração de instrumentos, são os argumentos que justificam tal estudo.

Considera-se que ao analisar o potencial da fotolinguagem e do photovoice a partir das dimensões teóricas das RS o artigo em tela traz inovação ao conhecimento. Além do que, estudos com instruções metodológicas à comunidade científica contribuem para o maior aprofundamento das análises de RS, o que possibilita avanços à pesquisa qualitativa na área da saúde e, em especial, à pesquisa em enfermagem, disciplina de inserção dos pesquisadores deste artigo.

Questiona-se: como as técnicas de produção de dados baseadas no uso de imagens por fotografias contribuem na captação das RS de fenômenos da área da saúde? Objetivo: refletir sobre o uso de fotos na apreensão de RS de fenômenos na área da saúde, a partir da aplicação do método do photovoice e da técnica da fotolinguagem.

\section{MÉTODO}

Estudo teórico-reflexivo desenvolvido em duas etapas. A Etapa 1 foi de revisão da literatura sobre o tema, em que se fez um levantamento exploratório on-line de artigos científicos que abordassem as técnicas de produção de dados nos estudos de RS, bem como sobre as possibilidades existentes de uso de imagens; e busca manual em uma biblioteca setorial de pós-graduação de livros de cunho teórico e metodológico sobre as RS e estratégias metodológicas de produção de dados. O objetivo dessa primeira etapa foi avaliar o estado da arte, analisando o conhecimento existente sobre fotos e RS. A partir dessa análise, construiu-se as duas primeiras seções deste artigo, de apresentação dos aspectos teóricos e metodológicos que sustentam a aplicação da fotolinguagem e do photovoice, como possibilidades de técnica e método de produção de dados nos estudos de RS.

Na Etapa 2, procedeu-se a análise reflexiva sobre a técnica da fotolinguagem e método do photovoice na interface com a perspectiva teórica das RS, o que subsidiou a organização da terceira seção do artigo. Para essa análise, foram aplicados os conceitos de zona muda das RS, dimensão afetiva e dimensão figurativa das RS, além da definição de triangulação metodológica e sua utilização na psicologia social. Esses conceitos foram empregados para evidenciar as potencialidades teóricas do uso 
de fotos na apreensão dos elementos que organizam RS sobre fenômenos no campo da saúde.

Para ilustrar os argumentos teóricos trazidos nas reflexões e demonstrar tal potencial de aplicação da fotolinguagem e photovoice na prática de pesquisa, foram trazidos os resultados de duas investigações prévias desenvolvidas pelos autores com a utilização dessas estratégias metodológicas.

\section{DESENVOLVIMENTO}

\section{A fotolinguagem: aspectos teóricos e metodológicos}

A fotolinguagem foi criada, em 1965, por psicólogos e psicossociólogos como uma ferramenta de estímulo à expressão discursiva de adolescentes com dificuldades para expressar suas experiências em grupo. $O$ resultado positivo habilitou sua utilização na formação de adultos, em empresas, na área social, para iniciar ou finalizar um grupo ${ }^{6}$.

No campo do cuidado clínico vem sendo empregada com função terapêutica na área psiquiátrica por psicólogos e enfermeiros com grupos de adolescentes, psicopatas, idosos e usuários de drogas. Nesse caso, o grupo tem em torno de oito participantes que se reúnem, semanalmente, por, aproximadamente, uma hora tendo como mote uma questão disparadora, que evolui com a continuidade do trabalho e o pensamento do grupo 6 .

A sessão é iniciada por uma questão elaborada pelo moderador e apresentada ao grupo que suscita a escolha das fotos. Tal questão requer atenção em sua proposição, pois não deve ser muito direta, longa e complexa. Quanto às fotos, são trazidas ao grupo sob a forma de dossiês temáticos com 48 fotos, cuja seleção é feita a partir de grupos prévios que levantam representações sobre o tema e, posteriormente, são pesquisadas as fotos, que são testadas em novo grupo, com a escolha daquelas mais significantes ${ }^{6}$.

A partir da questão, as fotos são dispostas sobre uma mesa e os participantes devem escolher a foto que é mais representativa para si. A escolha deve ocorrer de forma silenciosa, pelo olhar, para que todas as fotos permaneçam à disposição dos participantes, sem a troca da foto escolhida. Nos grupos clínicos, o moderador participa da escolha, pois isso, influencia na forma como é percebido pelos demais participantes ${ }^{6}$.

Posteriormente, ocorrem as trocas em grupos. Nesse momento, os participantes apresentam a sua foto e as explicações da sua escolha, e os demais dizem aquilo que veem de semelhante ou diferente da foto. Esse jogo de escuta e de compartilhamento da sua visão pessoal da realidade cria uma cadeia associativa de ideias que facilita as trocas identificatórias e a transformação das percepções/sentidos sobre a foto 6 .

A particularidade de solicitar a resposta a uma questão através de uma foto mobiliza o pensamento em imagens, que trazem em si imagens interiorizadas e os afetos que as acompanham, seguida do pensamento em ideias. Esse espaço intermediário entre o pensamento em imagens e em ideias, quando os participantes falam sobre a foto do outro, favorece a articulação entre o intrapsíquico e o intersubjetivo ${ }^{6}$.
A fotolinguagem vem sendo aplicada em estudos de RS, pois essas como conjuntos de conhecimentos organizados se enraízam no inconsciente, numa dimensão intrapsíquica e nas comunicações sociais, nas quais são compartilhadas crenças, valores, informações, atitudes ${ }^{9}$. Por enraizarem-se no inconsciente, é fundamental o uso de técnicas especiais para captar essas RS, como é o caso das projetivas. Tais técnicas têm a capacidade de acessar elementos enraizados no aparelho psíquico, tornando o inconsciente consciente ${ }^{9}$.

As técnicas projetivas são divididas em: temáticas, que favorecem aos indivíduos revelarem elementos de conflitos, desejos, reações ao meio ambiente, mecanismos de defesa. Como exemplos têm-se os desenhos, as associações livres de palavras, a interpretação de fotografias, o desenho história com tema; e estruturais, que não apropriam-se da manifestação viva da pessoa, mas como esta apreende a sua subjetividade, a exemplo do Roschach clássico ${ }^{9}$. Nessa perspectiva teórica, a fotolinguagem é considerada uma técnica projetiva temática que pode ser realizada, individualmente, quando a intenção é captar a dimensão espontânea da RS, que pode ser modificada com técnicas como as entrevistas individuais ou em grupo.

A imagem mediada pela foto é uma imagem sensorial, pois se inscreve na experiência corporal. Quando uma foto se impõe ao participante, Ihe toma, ela não é mais uma simples fotografia, mas uma imagem, ou seja, conota outra coisa para além dos dados objetivos que denota. Dessa feita, a foto media e transforma o imaginário, suporta as projeções, tem uma vertente visível, real, e uma metaforizante de outra realidade ${ }^{6}$.

\section{Photovoice: Aspectos teóricos e metodológicos}

A fotografia pode ser vista em estudos de pesquisa-ação como um instrumento catalisador de mudanças, justificado pela dupla função que a imagem fotográfica pode assumir, seja como expressão criativa visual ou como um meio de retratação de realidades e contextos. Nesse sentido, pode ser utilizada como instrumento que fornece aos pesquisadores sociais oportunidade de acessar distintas realidades, perspectivas e sensibilização de participantes para as questões sociais que os rodeiam ${ }^{10}$.

No que concerne ao Photovoice, é um método de produção fotográfica que tem sido difundido nas pesquisas sociais e na área da saúde, pois torna os sujeitos ativos na produção dos dados e construção da pesquisa. Esse tipo de pesquisa-ação permite ampliar o cenário e as possibilidades de intervenção para o participante e pesquisador, uma vez que retrata realidades. Além disso, as imagens, uma vez registradas, não podem ser silenciadas com as palavras e, ao serem compartilhadas e associadas às vivências e experiências, podem ressuscitar subjetividades alienadas pelo sistema social ${ }^{11,12}$.

Foi criado na década de 1990, por pesquisadoras da Universidade de Michigan e de Londres com foco na tríade: promoção da saúde, desenvolvimento comunitário e educação dialógico-crítica, pautando-se nos preceitos do educador Paulo Freire ${ }^{13}$. 
Consiste em uma estratégia participativa, na qual, pessoas usam câmeras para documentar suas realidades. $O$ foco central é acessar a visão de mundo de outras pessoas de forma mais próxima possível de suas realidades. As imagens e histórias associadas são desenvolvidas no diálogo em grupo, onde se compartilha experiências de mudanças das pessoas ${ }^{8}$. Esse processo grupal mediado pelas fotos promove um pensamento crítico, a partir do qual os participantes podem se tornar agentes de mudanças ${ }^{14}$.

No photovoice, a fotografia é o instrumento para "representação de perspectivas daqueles que levam uma vida diferente dos meios que, tradicionalmente, possuem o controle das imagens do mundo"15:154. A imagem fotografada pelos próprios envolvidos na pesquisa serve como uma alternativa ao registro escrito, o que, por si só, promove a inclusão dos participantes como informantes e até mesmo como pesquisadores ${ }^{10}$.

No que concerne a implementação e operacionalização do Photovoice, destacam-se os seguintes passos a serem adaptados de acordo com o contexto de cada estudo: 1 -fase do treinamento, na qual o pesquisador fornece câmeras e explica como utilizá-las, sanando dúvidas sem, contudo, limitar a liberdade dos participantes no momento da produção fotográfica; 2-realização das fotografias pelos participantes a partir de questão norteadora; 3-revelação das fotografias e devolutiva aos participantes. $O$ pesquisador pode fornecer cópias das imagens aos participantes, conforme acordo previamente estabelecido; 4-grupos focais de discussão, nos quais os participantes apresentam suas fotografias, atuando como catalisadores dos temas de reflexão e expressão de vivências e subjetividades ${ }^{13}$.

Nos grupos focais, os participantes são orientados a desenvolver as seguintes opções: Selecionar: escolher as fotografias que mais refletem as necessidades e recursos da comunidade ou grupo que se inserem; Contextualizar: discutir ou contar histórias ou experiências relacionadas aos significados das fotografias escolhidas; Codificar: identificar as questões, temas ou teorias que emergem nesse processo de discussão ${ }^{13}$.

\section{O uso de fotos para captar RS de fenômenos da área da saúde: uma reflexão}

A psicologia social tem dispensado pouca atenção ao uso das imagens, sejam estáticas (fotos) ou dinâmicas (filmes), como ferramentas de pesquisa na identificação da forma como as pessoas constroem a realidade social. Nesse entendimento, é preciso visibilizar o componente figurativo das $\mathrm{RS}^{16}$. Isso, porque, toda representação tem uma imagem objetivada, uma dimensão imagética do objeto social que o simplifica e que é naturalizada como o próprio objeto real. Dessa feita, a objetivação estabelece um núcleo de imagens que reflete um conjunto complexo de ideias ${ }^{1}$.

Esse papel simplificador da RS oculta a avaliação efetuada sobre o objeto social, o que pode mascarar valores que servem de referência para a construção do pensamento dos grupos sociais. Assim, a partir da objetivação, o objeto ganha valor de verdade e resiste às tentativas de questionamento dessa veracidade ${ }^{17}$.
Sob essa ótica, há conteúdos que podem ser mascarados pelos sujeitos da pesquisa, a zona muda da RS.

A zona muda são espaços de RS que não são expressas com facilidade nos discursos diários, pois são contranormativas, ou seja, não são adequadas às normas sociais vigentes. Há, portanto, o que os teóricos chamam de "representações mascaradas", nas quais os elementos que podem entrar em conflito com os valores morais dos grupos ficam escondidos ${ }^{18}$.

Logo, no âmbito da investigação em RS, parte-se do pressuposto de que nem sempre as pessoas investigadas dizem o que realmente pensam, sendo relevante acessar esse não dito. Na área da saúde, estudos têm apontado a zona muda em relação a fenômenos sensíveis, como é o caso do envelhecimento/rejuvenescimento, cujo discurso normativo sobre o envelhecimento natural e saudável pode mascarar cognições relacionadas ao medo de envelhecer e as práticas adotadas para o rejuvenescimento; ou do HIV/Aids para profissionais de saúde, em que os termos medo e preconceito evocados possuem um caráter contranormativo e podem ser constitutivos da zona muda ${ }^{19,20}$.

O estudo clássico sobre as RS da loucura é também ilustrativo sobre como práticas sociais expressam crenças, acessíveis por manifestações verbais, mas também icônicas, comportamentais, gestuais, rituais, rotineiras, práticas ou artísticas ${ }^{21}$.

Esses aspectos teóricos justificam o uso de fotos, escolhidas ou produzidas, pois oportunizam compreender o indizível das RS pela captação da sua dimensão icônica. O potencial das fotos na captação da zona muda pôde ser visto com a aplicação do photovoice na pesquisa com 15 adolescentes sobre as suas RS de ser saudável. Nessa pesquisa prévia desenvolvida por dois dos autores deste artigo, partiu-se da premissa teórica de que existiam aspectos da RS desse objeto (ser saudável na adolescência) que se inscreviam na zona muda, porque abarcava temas mais sensíveis e marcados por valores e normas sociais, como sexualidade e uso de drogas ${ }^{22}$.

Durante a aplicação da etapa do photovoice, os adolescentes foram divididos em dois grupos com idade média entre $11 \mathrm{e}$ 14 anos para a realização das atividades fotográficas, seguida da etapa de discussão nos grupos focais ampliados ${ }^{22}$.

O uso do photovoice fomentou a compreensão do fenômeno ser saudável na adolescência para além de um discurso politicamente correto pautado nos conceitos científicos da manutenção da saúde, bem como nos saberes compartilhados com a família. A partir das fotografias e da negociação em grupo, emergiram as práticas sociais, nas quais as relações de amizade e de grupo orientaram as atitudes frente a sua saúde ${ }^{22}$.

Nos cuidados com o corpo sobressaiu o culto do corpo perfeito, percebendo-se a falta de informação quanto as consequências à saúde a longo prazo das atitudes tomadas para a sua obtenção. Tal imagem corporal foi construída com base nas representações que circulam nas mídias sociais, de fácil acesso para o grupo pela internet. Observou-se maior insatisfação nas mulheres, com ideias mais distorcidas da adequação do peso à altura, e as que mais fizeram dieta para emagrecer. Sobre as atividades 
físicas, houve maior envolvimento por parte dos homens, pelo caráter de masculinidade balizado na definição e tamanho de massa muscular ${ }^{22}$.

Quanto ao envolvimento dos adolescentes com a alimentação saudável e com níveis de atividades físicas adequados a sua idade, esteve longe do desejado para uma vida saudável e para o combate à obesidade. A inatividade física foi preocupante nos participantes, que preferiam assistir televisão e utilizar o computador e/ou videogame ${ }^{22}$.

No consumo de álcool, mulheres e homens o consomem em situações festivas e entre amigos, não havendo limites para a sua interrupção, sendo os destilados preferidos pelas mulheres e a cerveja pelos homens. Os comportamentos sexuais, por sua vez, revelaram a falta de informação frente aos riscos da não proteção e, apesar de a grande maioria ainda não ter tido a primeira relação sexual, a preocupação foi com a gravidez ${ }^{22}$.

A segunda possibilidade do uso das imagens é a de considerar o seu papel na captação das emoções. A dimensão afetiva é a base da familiaridade com o grupo de pertença social. Assim, quando um objeto provoca tal grupo, os sujeitos que com ele se identificam querem participar do circuito de conversação mobilizado pelo objeto, para se sentirem incluídos, serem reconhecidos, conseguirem se comunicar a partir de uma linguagem comum. Nesse entendimento, os afetos são essenciais à elaboração das RS a partir desta conversação, a qual organiza cognições ou comportamentos avaliativos ${ }^{23}$.

As técnicas projetivas possibilitam captar esses afetos, uma vez que com o seu uso considera-se que perguntas e respostas não são diretas, entram no campo metafórico. A metáfora, em vez de dizer tudo, revela enquanto esconde, referindo-se ao inconsciente e de cunho afetivo-emocional. São indicadas quando indivíduos possuem problemas na esfera física e/ou psicológica (medo, rejeição, morte, tristeza, autoimagem) ${ }^{9}$.

$\mathrm{Na}$ fotolinguagem, uma dessas técnicas projetivas, a foto como objeto mediador se torna uma imagem que mobiliza imagens interiores associadas e religadas pelo afeto que as sustenta, contribuindo para ampliação das atividades pré-conscientes ${ }^{6}$. Um exemplo do potencial da fotolinguagem foi evidenciado em uma pesquisa que demonstrou o papel das imagens na construção da memória social frente a objetos culturais através do filtro das emoções e sistemas de representações. Memória social entendida como um tipo de estudo que busca descobrir nem tanto o quanto é lembrado de um acontecimento, mas o que é lembrado e como a memória é construída socialmente, quais filtros ideológicos são empregados, que emoções são evocadas, os interlocutores envolvidos ${ }^{16}$.

O estudo centrou-se no processo de memória de um grande acontecimento social, repleto de comunicações e imagens, o ataque às Torres Gêmeas em 11 de Setembro de 2001, tendo sido abordados 522 participantes ${ }^{16}$.

Os sujeitos responderam um questionário sobre imagens mentais para lembrar/esquecer; e selecionaram imagens em grupos de fotolinguagem a partir de fotos mais usadas na mídia, para identificar imagens que causaram uma impressão mais forte no primeiro momento, negociadas no grupo, e imagens mais representativas ${ }^{16}$

As emoções tiveram um papel central na seleção das imagens. Os autores perceberam que na seleção das cinco imagens mais lembradas e mais representativas houve uma sequência normativa linear centrada nas mesmas imagens (choque dos aviões com as torres). Já no caso das cinco imagens para esquecer e as mais impressionantes, no lugar do choque dos aviões apareceram as imagens das vítimas (presas nas torres, saltando para se salvarem), de onde emergiram emoções como: desespero, angústia, tristeza, horror ${ }^{16}$.

Confirmou-se o papel das emoções na construção seletiva da memória e, com isso, concluiu-se sobre a importância de se estudar o componente icônico das RS e o seu impacto emocional, realçando as implicações metodológicas dessa escolha teórica ${ }^{16}$

Na área da saúde, vários objetos de RS são revestidos dessa carga afetiva, que mobiliza atitudes, como a hanseníase, marcada pelo estigma que gera o embotamento social dos portadores; a eutanásia, que mobiliza o debate no campo da saúde, jurídico e da religião a partir de valores e crenças sociais; e o corpo mastectomizado para as mulheres, que associa representações negativas pela aparência da mama $24-26$

O potencial da fotolinguagem para captação dessa dimensão afetiva, essencial no processo de elaboração das RS, pode ser visualizado no estudo com portadores de úlceras vasculogênicas acerca da sua qualidade de vida (QV), realizado por autores deste artigo. A pesquisa foi desenvolvida com 30 pacientes de uma Clínica da Família com úlceras vasculares há mais de três meses. A escolha da fotolinguagem partiu do pressuposto de que a lesão vasculogênica remete a uma dimensão imagética sobre o corpo, que traz à tona identidades e afetos ${ }^{27}$.

A técnica consistiu em mostrar fotografias cromáticas aos pacientes que se relacionavam a elementos que ilustravam o cotidiano e tinham nexos com a QV. A questão indutora foi: como é a sua vida tendo que conviver com uma ferida na perna? Foi fornecido um tempo para que o paciente pudesse proceder essa escolha e, em seguida, procedeu-se aos seguintes questionamentos: esta imagem é positiva ou negativa? O que ela expressa para você ${ }^{27}$.

Os dados mostraram que o fenômeno estava imerso numa dualidade afetiva. Isso, porque, quando os pacientes foram questionados acerca da conotação afetiva que a imagem escolhida expressava em relação a sua vida com uma úlcera, no primeiro momento, grande parte associou as imagens aos afetos positivos, o que revelou um aspecto científico normativo, particularmente, nas imagens que aludiam às práticas alimentares e de atividade física. Todavia, nas explicações emergiu a forma como o científico é ressignificado no social, ou seja, as emoções e sentimentos negativos pelas perdas advindas da nova vida com a úlcera ${ }^{27}$.

A imagem mais escolhida entre os 30 participantes foi a que trazia duas cestas de verduras e frutas, cuja explicação as relacionavam a uma "boa alimentação/alimentação saudável" que ajudava na cicatrização da ferida, prática que buscavam adotar com base nas orientações feitas pelos profissionais de 
saúde. Por outro lado, preocupavam-se em não comer alimentos que lhes prejudicassem e, como consequência, adotar um novo estilo de vida pelas mudanças alimentares era algo difícil de lidar e de implementar ${ }^{27}$.

Outra imagem escolhida foi a que trazia um grupo de pessoas se exercitando em um local público. Os pacientes veicularam, inicialmente, a ideia do exercício físico como importante pelos benefícios que traz para a saúde. Todavia, como nem sempre era possível realizá-lo, se lembravam da sua capacidade física do passado e o desejo de resgatá-la. Esse retorno em relação à capacidade funcional antes do surgimento da úlcera, trouxe à tona as perdas em relação à prática de atividades como jogar bola, caminhada, ginástica, correr. Nesse sentido, embora a atividade física em si fosse positiva, quando se reportavam ao presente surgiam os afetos negativos por não poderem realizá-la ${ }^{27}$.

A análise dos dados mostrou que os pacientes utilizavam expressões como: "não posso mais fazer"; "eu gostaria de voltar a fazer"; "tive que banir"; para se remeterem às mudanças para um novo estilo de vida, mencionando o passado em relação à vivência do presente. Logo, os afetos que emergiram dessas mudanças, pelas perdas vivenciadas em relação a si, na manutenção do cuidado consigo, e sociais foram orientadores das RS sobre a QV e das práticas a ela articuladas, o que foi captado pela técnica projetiva.

A terceira possibilidade de uso das imagens nos estudos de RS é a de triangulação, que ganhou força na psicologia social a partir do advento do paradigma socioconstrucionista. A triangulação significa validar o conhecimento produzido pelos cruzamentos de resultados obtidos por diferentes métodos/técnicas ${ }^{28}$.

A psicologia social da saúde, na qual importa o sujeito e sua inscrição social (macrocontexto) e também as interações estabelecidas no seu microcontexto tem se desenvolvido nessa ótica. Logo, essa contextualização na compreensão dos fenômenos da saúde demanda um polimorfismo metodológico ${ }^{28}$. O uso das fotos pode ser então uma estratégia metodológica complementar nas pesquisas de RS, buscando-se captar a dimensão espontânea do fenômeno investigado, os afetos investidos, enquanto que outras dimensões das RS podem ser aprofundadas por meio de técnicas como a entrevista e a observação. Com isso, permite-se o melhor entendimento dos elementos que organizam o campo das RS, aspecto relevante de ser demonstrado nos estudos que se utilizam da abordagem processual das RS.

O potencial da triangulação para compreensão do macrocontexto e microcontexto implicados na construção das RS de fenômenos no campo da saúde é exemplificado no estudo que investigou como as RS influenciam nas comunicações e nas práticas relacionadas à tomada de decisão dos médicos oncologistas ${ }^{28}$.

Os autores combinaram estratégias qualitativas e quantitativas: 1. Triangulação na coleta -imersão em campo para apreender as categorias naturais exibidas pelos médicos em situações reais de tomada de decisão, usadas em conjunto com a literatura para construir o guia da observação; observação com o guia; entrevista, com roteiro construído com base na observação e nos aspectos teóricos; 2 . Triangulação na análise - as falas dos médicos durante as reuniões foram codificadas, com análise estatística da frequência com que características não médicas apareciam nas discussões coletivas; análise qualitativa para apreensão global do fenômeno; análise temática das entrevistas complementada pelo software Nvivo. Houve, ainda, a seleção de uma entrevista para análise fenomenológica interpretativa, método que tem raiz epistemológica comum às RS; 3. Triangulação de pesquisadores - pesquisadores de diferentes áreas participaram da codificação das observações e na verificação da confiabilidade interexaminadores na codificação, bem como na fase de análise temática ${ }^{28}$.

A última fase foi de cruzamento dos resultados, qualitativos e quantitativos. A triangulação possibilitou entender diversos aspectos do fenômeno (triangulação da coleta), forneceu confirmação dos dados (triangulação da análise) e plausibilidade das análises (triangulação dos pesquisadores), refinando a compreensão das RS do fenômeno ${ }^{28}$.

Na pesquisa sobre as RS da QV por pacientes com úlceras vasculogênicas também houve triangulação na coleta, pois a fotolinguagem foi utilizada em conjunto com entrevistas semiestruturadas, e da análise, com o emprego da análise de conteúdo temático (fotolinguagem) e do software Alceste para processamento das entrevistas. Essa triangulação possibilitou aprofundar as dimensões da informação e práticas sociais por meio das entrevistas que, complementadas pelos afetos e atitudes que emergiram da fotolinguagem, delimitaram o campo de organização das RS sobre a QV²7.

Por fim, à luz do caráter figurativo das RS, ressaltam-se as associações entre figura/significação e o potencial das imagens nos estudos de RS. As RS permitem interpretar as imagens do mundo e produzir imagens sociais do mundo. Tal compreensão reenvia a três níveis de análise: perceptivo, cognitivo e simbólico ${ }^{29}$.

No perceptivo, o suporte imagético possui três propriedades: a de figuração, de substituir um determinado objeto; sua capacidade de gerar emoções; e sua ambiguidade, isto é, o sentido da imagem é apropriado pelo indivíduo que a percebe. No nível cognitivo, a imagem torna-se uma imagem mental, não como uma simples imagem visual, mas com uma capacidade de se associar a forma (significante) a um sentido (significado). No caso da imagem mental, combinam-se elementos figurativos com semânticos, significados por palavras. No nível simbólico, certas palavras têm capacidade de suscitar imagens. Assim, a correspondência da palavra a uma imagem mental opera um processo simbólico ${ }^{29}$

Conclui-se, que RS podem se exprimir sob a forma de imagens mentais que dão efeito de concretude ao objeto. A ativação de múltiplas imagens mentais resulta na imagem social do objeto. Nesta ótica, é fundamental recolher conteúdos ligados às imagens a partir da seleção ou expressão de imagens visuais ${ }^{29}$.

\section{CONCLUSÃO E IMPLICAÇÕES PARA A PRÁTICA}

O uso de fotos nos estudos de RS potencializa apreender a sua dimensão figurativa, o que é essencial para se compreender os processos de elaboração do pensamento social. A reflexão em tela apontou alguns elementos teóricos que fundamentam a 
construção de RS passíveis de serem acessados pela fotolinguagem e photovoice: a zona muda das RS, a dimensão dos afetos, a dimensão simbólica, e o entendimento ampliado do contexto que concorre para a organização do campo da representação de fenômenos no campo da saúde, considerando a aplicação das fotos como uma das estratégias metodológicas de triangulação.

Conclui-se que a fotolinguagem e o photovoice se fundam em princípios diferentes, mas convergem como possibilidades metodológicas nos estudos de RS, escolha que deve ser guiada por preceitos teóricos. O uso dessas estratégias na prática operacional de pesquisa contribui para o avanço do conhecimento nos estudos de RS na área da saúde.

As limitações são teóricas, relacionadas à abrangência do referencial das RS como uma das possibilidades de análise das potencialidades da técnica e método discutidos no artigo, dentre outras existentes.

\section{CONTRIBUIÇÕES DOS AUTORES}

Desenho do estudo de reflexão. Nathália Nunes Gomes Julyana Gall da Silva. Ana Beatriz Azevedo Queiroz. Márcia de Assunção Ferreira. Thémis Apostolidis. Rafael Celestino da Silva.

Aquisição de textos. Nathália Nunes Gomes. Julyana Gall da Silva. Ana Beatriz Azevedo Queiroz. Márcia de Assunção Ferreira. Thémis Apostolidis. Rafael Celestino da Silva.

Análise teórico-reflexiva. Nathália Nunes Gomes. Julyana Gall da Silva. Ana Beatriz Azevedo Queiroz. Márcia de Assunção Ferreira. Thémis Apostolidis. Rafael Celestino da Silva.

Interpretação dos resultados. Nathália Nunes Gomes. Julyana Gall da Silva. Ana Beatriz Azevedo Queiroz. Márcia de Assunção Ferreira. Thémis Apostolidis. Rafael Celestino da Silva.

Redação e revisão crítica do manuscrito. Nathália Nunes Gomes. Julyana Gall da Silva. Ana Beatriz Azevedo Queiroz. Márcia de Assunção Ferreira. Thémis Apostolidis. Rafael Celestino da Silva.

Aprovação da versão final do artigo. Nathália Nunes Gomes. Julyana Gall da Silva. Ana Beatriz Azevedo Queiroz. Márcia de Assunção Ferreira. Thémis Apostolidis. Rafael Celestino da Silva

Responsabilidade por todos os aspectos do conteúdo e a integridade do artigo publicado. Nathália Nunes Gomes. Julyana Gall da Silva. Ana Beatriz Azevedo Queiroz. Márcia de Assunção Ferreira. Thémis Apostolidis. Rafael Celestino da Silva.

\section{EDITOR ASSOCIADO}

Eliane Tatsch Neves (D)

\section{EDITOR CIENTÍFICO}

Ivone Evangelista Cabral (1)

\section{REFERÊNCIAS}

1. Moscovici S. Representações sociais: investigações em psicologia social. Petrópolis (RJ): Editora Vozes; 2015.
2. Felippe ML, Kuhnen A, Da Silveira BB, Klein C. Realidade mediada: compreendendo qualidades restauradoras de ambientes através da fotografia. Psi Saber Soc. 2017;6(1):26-41. http://dx.doi.org/10.12957/ psi.saber.soc.2017.25083.

3. Terra IG, Nascimento ARA. Images and social representation: semiotic analysis contributions. Psicol Estud. 2016;21(2):291-302. http://dx.doi. org/10.4025/psicolestud.v21i2.29783.

4. Almeida RO, Oliveira FT, Ferreira MA, Silva RC. Newly undergraduate nurses and intensive care in units of non-critical patients. Rev Bras Enferm. 2019;72(Suppl 1):243-51. http://dx.doi.org/10.1590/00347167-2017-0713. PMid:30942369.

5. Silva JG, Ferreira MA. Diet and health in the perspective of adolescents: contributions for health promotion. Texto Contexto Enferm. 2019;28:e20180072. http://dx.doi.org/10.1590/1980-265x-tce-2018-0072.

6. Vacheret CA. A Fotolinguagem $\odot$ : um método grupal com perspectiva terapêutica ou formativa. Psicol Teor Prat [Internet]. 2008 [citado 2019 ago 8]; 10(2):180-91. Disponível em: http://pepsic.bvsalud.org/pdf/ptp/ v10n2/v10n2a14.pdf

7. Brito TDQ, Oliveira AR, Eulálio MC. Deficiência física e envelhecimento: estudo das representações sociais de idosos sob reabilitação fisioterápica. Av en Psicol Latinoam. 2015;33(1):121-33. http://dx.doi.org/10.12804/ apl33.01.2015.09.

8. Fernandes CS, Carvalho JN, Feitora ES, Costa NP, Moraes TM, Rodrigues ARS et al. Photovoice as a research method in health education: an integrative review. Rev Eletr Enferm. 2019;21:1-18. http:// dx.doi.org/10.5216/ree.v21.51434.

9. Coutinho MPL, Nóbrega SM, Catão MFFM. Contribuições teóricometodológicas acerca do uso dos instrumentos projetivos no campo das Representações Sociais. In: Coutinho MPL, Lima AS, Oliveira FB, Fortunato M, organizadores. Representações sociais: abordagem Interdisciplinar. João Pessoa: Editora Universitária; 2003. p. 50-66.

10. Meirinho D. O olhar por diferentes lentes: o photovoice como método científico participativo. Discursos fotog. 2017;13(23):261-90. http:// dx.doi.org/10.5433/1984-7939.2017v13n23p261.

11. Touso MFS, Mainegra AB, Martins CHG, Figueiredo GLA. Photovoice como modo de escuta: subsídios para a promoção da equidade. Cien Saude Colet. 2017 dez;22(12):3883-92. http://dx.doi.org/10.1590/1413812320172212.25022017. PMid:29267706.

12. Guillemin M, Drew S. Questions of process in participant-generated visual methodologies. Vis Stud. 2010;25(2):175-88. http://dx.doi. org/10.1080/1472586X.2010.502676.

13. Wang C, Burris MA. Photovoice: concepts, methodology and use for participatory needs assessment. Health Educ Behav. 1997;24(3):36987. http://dx.doi.org/10.1177/109019819702400309. PMid:9158980.

14. Andina-Díaz E. Using Photovoice to stimulate critical thinking: an exploratory study with Nursing students. Rev Lat Am Enfermagem. 2020;28:e3314. http://dx.doi.org/10.1590/1518-8345.3625.3314. PMid:32609267.

15. Wang CC. Youth participation in photovoice as a strategy for community change. J Community Pract. 2006;14(1-2):147-61. http://dx.doi. org/10.1300/J125v14n01_09.

16. De Rosa AS. O impacto das imagens e do compartilhamento das emoções na construção da memória social: uma chocante memória flash de massa do 11 de setembro até a guerra do Iraque. In: Sá CP, organizador. Memória, imaginário e representações sociais. Rio de Janeiro: Museu da República; 2005. p. 121-64.

17. Medina AL Fo. Importância das imagens na metodologia de pesquisa em psicologia social. Psicol Soc. 2013;25(2):263-71. http://dx.doi. org/10.1590/S0102-71822013000200003.

18. Danfá L, Aléssio RLS, Santos MFS, Morais ERC. Preconceito e descontextualização normativa: considerações metodológicas ilustradas pelas representações sobre AIDS na África e Africano. Psychologica. 2017;60(2):83-99. http://dx.doi.org/10.14195/1647-8606_60-2_5.

19. Castro A, Giacomozzi Al, Camargo BV. Representações sociais, zona muda e práticas sociais femininas sobre envelhecimento $e$ rejuvenescimento. Estud Interdiscip Psicol. 2019;9(2):58-77. https:// doi.org/10.5433/2236-6407.2016v9n1p58 
20. Machado YY, Oliveira DC, Nogueira VPF, Gomes AMT. Health personnel's social representations of HIV/AIDS: a structural analysis. Rev enferm UERJ. 2016;24(1):e14463. https://doi.org/10.12957/reuerj.2016.14463.

21. Jodelet $D$. Ciências sociais e representações: estudo dos fenômenos representativos e processos sociais, do local ao global. Soc Estado. 2018;33(2):423-42. http://dx.doi.org/10.1590/s0102-699220183302007.

22. Silva JG. Ser saudável da adolescência: a busca de sentidos e implicações nas práticas de cuidado à saúde [tese]. Rio de Janeiro (RJ): Escola de Enfermagem Anna Nery, Universidade Federal do Rio de Janeiro; 2018.

23. Arruda A. Meandros da teoria: a dimensão afetiva das representações sociais. In: Almeida AMO, Jodelet D, organizadores. Interdisciplinaridade e diversidade de paradigmas. Brasília (DF): Thesaurus, 2009. p. 83-102.

24. Neiva RJ, Grisotti M. Representações do estigma da hanseníase nas mulheres do Vale do Jequitinhonha-MG. Physis. 2019;29(1):e2901092019. http://dx.doi.org/10.1590/s0103-73312019290109.

25. Santos JLF, Morais ERC, Aléssio RLS. Social representations on Euthanasia between students of law and medicine: a comparative analysis. Trends Psychol. 2019;27(3):805-18. http://dx.doi.org/10.9788/ tp2019.3-15.

26. Hirschle TMR, Maciel SC, Amorim GK. Social representations of the body and sexual satisfaction in mastecomized women and their partners. Trends Psychol. 2018;26(1):457-68. http://dx.doi.org/10.9788/tp2018.1. 18 pt.

27. Gomes, NN. Representações sociais de pacientes portadores de úlceras vasculogênicas sobre qualidade de vida [dissertação]. Rio de Janeiro (RJ): Escola de Enfermagem Anna Nery, Universidade Federal do Rio de Janeiro; 2019.

28. Restivo L, Apostolidis T. Triangulating qualitative approaches within mixed methods designs: a theory-driven proposal based on a French research in social health psychology. Qual Res Psychol. 2019;16(3):392-416. http://dx.doi.org/10.1080/14780887.2019.16 05670.

29. Moliner P. Représentations sociales et iconographie. Communication \& Organisation. 2008;34:12-23. https://doi.org/10.4000/ communicationorganisation.547. 